\title{
What do we still need to understand to commercialize cellulose nanomaterials? ${ }^{\dagger}$
}

1 Chelsea S. Davis

Materials Measurement Laboratory, National Institute of Standards and Technology, Gaithersburg, MD, USA

2 Danielle L. Grolman

Department of Polymer Engineering, University of Akron, Akron, $\mathrm{OH}$, USA
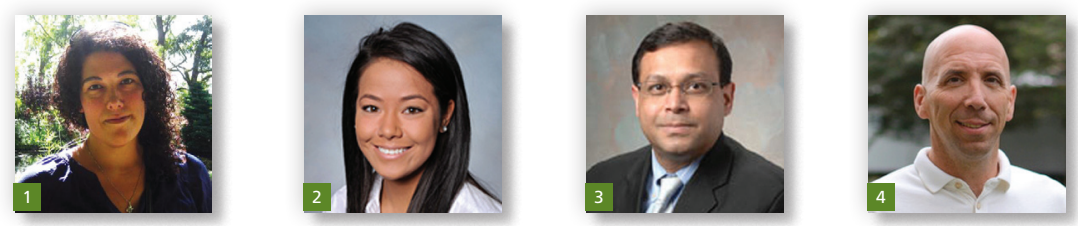

Cellulose nanomaterials (CNM) have the potential to become predominant nanoparticles utilized in materials engineering fields ranging from flexible electronic supports and tissue scaffolds to nanocomposite reinforcement materials and aerogels for oil capture. The potential of this emerging technological material is currently hindered by the inability efficiently to produce consistent cellulose nanomaterial-based products on an industrial scale. To quantify key properties of CNM (most notably surface properties, particle size and degree of crystallinity), several measurement methods and techniques have been employed by researchers. However, the currently available measurement techniques are too expensive (in terms of both time and money) to be applicable for high-throughput, full-scale industrial process monitoring. In an effort to highlight and identify the most critical measurement requirements, a workshop was conducted by National Institute of Standards and Technology in conjunction with the Technical Association of the Pulp and Paper Industry's Nanotechnology Conference in 2014. This workshop brought together key researchers and manufacturers at the forefront of CNM research and production. The goal of the workshop was to discuss the existing deficiencies in CNM metrology, as well as to inform and inspire measurement science development in the field.

\section{What are cellulose nanomaterials} and why are they of interest?

Cellulose nanomaterials (CNM) are naturally occurring nanoparticles present in lignocellulosic biomass (e.g., trees, grasses, municipal waste), bacteria (e.g., acetobacter) and invertebrate sea creatures (e.g., tunicates). Over the past decade, numerous applications for CNM have been developed and investigated worldwide..$^{1-10}$ Cellulose nanocrystals (CNC) were first isolated in the laboratory by Mukherjee and Woods in $1953,{ }^{11}$ and a commercially scalable process for cellulose nanofiber (CNF) production was invented by ITT Rayonier in 1977..$^{12,13}$ Since then, several inventions and techniques have led to the development of pilot-scale production methods which have been implemented across the globe (CelluForce in Canada, American Process, Inc. in the United States of America and Innventia in Sweden to name a few). CNM are an attractive alternative to other high-aspect-ratio nanoparticles, such as carbon nanotubes and silica nanowhiskers, due to their renewable, sustainable origins and expected low toxicity. (Toxicology studies are ongoing but 'powdered cellulose' has recently been placed on the list of Permitted Food Additives in Canada. ${ }^{14}$ )

Cellulose consists of glucose molecules linked together into polysaccharide chains, which align to form nanocrystalline domains. The hydroxyl substituent groups on each repeat unit form both inter- and intra-chain hydrogen bonds, leading to unique mechanical and thermal properties. ${ }^{7,15}$ The nanocrystalline domains are linked together into nanofiber bundles by flexible, amorphous hemicellulose regions. Various processing techniques have been developed to isolate the two general classes of CNM - that is, cellulose nanofibrils and the individual $\mathrm{CNC}$. Isolation of $\mathrm{CNC}$ is

*Corresponding author e-mail address: jeffrey.gilman@nist.gov

Official contribution of the National Institute of Standards and Technology (NIST); not subject to copyright in the United States. Description of commercial products herein is for information only; it does not imply recommendation or endorsement by NIST. 
typically accomplished through chemical processes utilizing acid hydrolysis ${ }^{11,16}$ or 2,2,6,6-tetramethylpiperidine-1-oxyl-mediated techniques. ${ }^{17} \mathrm{CNF}$ is often obtained by mechanical processing. ${ }^{7,18}$

Both $\mathrm{CNF}$ and $\mathrm{CNC}$ have diameters that typically range from 5 to $10 \mathrm{~nm}$. CNF are comprised of flexible, spaghetti-like particles that can reach lengths of $1 \mu \mathrm{m}$ or greater while CNC are straight, rod-like particles with lengths of the order of 50 to $1000 \mathrm{~nm}$, depending on the source and extraction method. A representative atomic force microscope image of wood-derived $\mathrm{CNC}$ is provided in Figure 1. CNM are of interest to materials researchers and product developers due to their unique materials properties. The morphology of the aligned cellulose chains within CNM result in high axial elastic modulus values $\left(\mathrm{E}_{\mathrm{CNF}}: 120-270 \mathrm{GPa}, \mathrm{E}_{\mathrm{CNC}}\right.$ : 105-180 GPa). ${ }^{19}$

Additional advantageous properties such as high strength-to-weight ratio, ${ }^{20}$ high aspect ratio, ${ }^{21}$ high stiffness ${ }^{22}$ and low coefficient of thermal expansion ${ }^{23}$ all make CNM an ideal nanoreinforcement in polymeric nanocomposite systems. In their neat form, CNM have optical properties which result in transparent films or papers. ${ }^{1,3}$ Under specific circumstances, CNC self-assemble into a chiral nematic crystalline order, thus having the potential to impart structural color to coated objects..$^{24,25}$ The unique surface properties of CNC, particularly, have been investigated both theoretically and experimentally. ${ }^{15}$

In addition to the exceptional materials properties that make CNM a good candidate material for many emerging engineering applications, the natural abundance and sustainable/renewable nature of CNM are particularly attractive in a world looking towards more environmentally friendly technological materials solutions. Cellulose is the most abundant biopolymer on the planet, as it comprises the bulk of all plant cell walls and is also found in certain bacteria and other non-plant organisms. ${ }^{4,5,26}$ Additionally, microfibrillated cellulose is already widely used in a vast array of consumer products including pharmaceutical tablets, food additives and thickeners. Although toxicological studies are ongoing, it is believed that $\mathrm{CNM}$ will prove to be inherently nontoxic, ${ }^{27}$ paving the way to acceptance by regulatory agencies for large scale industrial production and commercialization.

\section{Barriers to commercialization}

Currently, two major factors stand in the way of mass-producing CNM. The first and most immediate barrier is the absence of highthroughput, high-resolution process monitoring techniques for quality control and assurance. These methods are needed not only to ensure that products are being made consistently, but also to aid the development of more energy-efficient manufacturing methods. The second hurdle slowing down mass CNM commercialization is the delay in approval of CNM as a safe material (both environmentally and toxicologically) by federal and international regulatory agencies.

As highlighted at the National Institute of Standards and Technology (NIST) Workshop on Measurement Needs for Cellulose Nanomaterials, ${ }^{28}$ the ability to characterize key CNM properties quickly and reliably is critical to manufacture CNM reproducibly with consistent particle geometry, surface properties and molecular-scale morphology. The measurement techniques that are used by academics and researchers on the benchtop are too time intensive and require a high level of skill to operate - for example, electron microscopy and atomic force microscopy. Several pilotscale producers of CNM have called for the development of in-line process control measurement systems that would monitor key CNM properties.

With regard to regulatory barriers to the industrialization of CNM, the Nanotechnology Division of the Technical Association of the Pulp and Paper Industry (TAPPI) released a report in 2011 outlining a plan for the development of cellulose nanomaterial standards. ${ }^{29}$ The National Research Council of Canada is leading the charge to determine toxicity and develop reference materials for both CNC and CNF. In fact, the list of Permitted Food Additives on the Health Canada website was updated in 2014 to include 'powdered cellulose' as a bulking agent for baked goods. ${ }^{14}$ Reference materials are already available from the NRC-Canada which include woodderived $\mathrm{CNC}$ powder (CNC-1) and $\mathrm{CNC}$ in aqueous suspension (CNCS-1). The Canadian Standards Association (CSA) has released CSA Z5100-14 'Cellulosic Nanomaterials - Test Methods of Characterization' which details a battery of test methods related to the characterization and identification of $\mathrm{CNM} .{ }^{30}$ Manufacturing

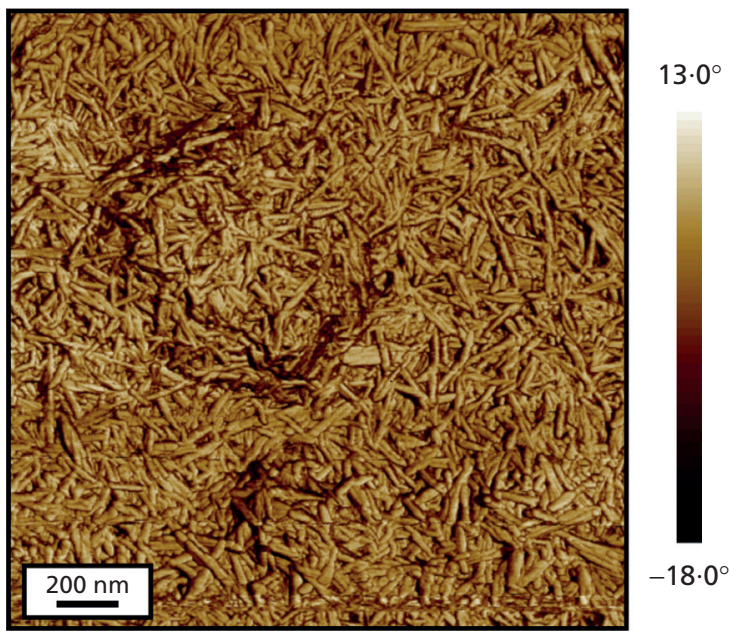

Figure 1. Representative atomic force microscope (AFM) phase contrast image of concentrated mat of wood-sulfate CNC. The average CNC length and width are $130 \pm 67 \mathrm{~nm}$ and $5 \cdot 9 \pm 1 \cdot 8 \mathrm{~nm}$, respectively 
standards are also being developed by several other international organizations (International Standards Organization Technical Committee 229 and ASTM International E-56).

\section{NIST Cellulose Nanomaterial Workshop}

A one-day workshop focused on the Measurement Needs for Cellulosic Nanomaterials was organized by the National Institute of Standards and Technology and held in conjunction with the 2014 TAPPI International Conference on Nanotechnology for Renewable Materials in Vancouver, British Columbia, Canada, on June 23, 2014. The workshop consisted of presentations by leaders from industry, academia and standards policy groups who work with CNM. In addition, three breakout sessions, focused on manufacturing, research and development, and standards development, were held to allow for more pointed and in-depth discussion of specific measurement needs within each discipline.

The outcome of the workshop clearly identified barriers to innovation related to a number of significant measurement challenges. The critical measurement needs for processing cellulose nanomaterial were sorted into four characterization categories: (a) geometry, (b) chemistry, (c) processing and (d) environmental health impacts. The most critical geometry measurement needs were fast, nano-scale resolution of particle size and morphology. In particular, the degree of branching and/ or fibrillation for $\mathrm{CNF}$ and aspect ratio for $\mathrm{CNC}$ must be assessed. Chemistry characterization was divided into surface chemistry (surface energy, surface functionalization, charge density along the length, etc.) and internal structure (degree of crystallinity, organization of amorphous chains, polymer chain morphology, etc.). Processing needs included measurements of aggregation and redispersion, processing-structure-property relationships for composites and durability (service life). Environmental health measurement needs were focused on quantitative detection of CNM in liquids and in air. These measurements are critical to enable the development of environmental health and safety standards for CNM commercialization.

The participants representing the manufacturing sector agreed that the greatest measurement needs are high-throughput characterization of CNM geometry (size and morphology), surface energy and surface chemistry. Most of the current analytical techniques (scanning electron microscopy, transmission electron microscopy, etc.) used to quantify these key properties are too expensive (in terms of operator training, acquisition speed and capital cost) for quality control in a manufacturing environment. Additionally, any new, faster techniques that are developed must still maintain a sufficient level of sensitivity and resolution to detect changes in CNM properties during production.

The research and development discussion focused on three major commercial applications of CNM: nanocomposite systems, viscosity modifiers and thin film barrier applications. To enable science-based advancement of these CNM applications, researchers were in agreement with the manufacturing sector, stating that the most critical measurement needs are characterization of geometry and chemistry. These properties are essential for both manufacturing a consistent product and exploiting these properties for innovation within the application space.

Finally, the standards breakout session identified a three-pronged approach to CNM commercialization. First, the development of standard test methods for key CNM properties (primarily morphology and chemistry) that enable consistency and quality control in manufacturing. Second, environment, health and safety studies focused on CNM toxicity (for both manufacturing and commercial product release) as well as environmental impact prior to large-scale production. Finally, reference materials, such as those offered by NRC-Canada, must be utilized to facilitate collaboration between academia and industry.

Currently, international efforts are underway in both the public and private sectors to develop new applications for CNM and to manufacture CNM as efficiently as possible. This workshop was a step towards understanding the measurement needs of this growing industrial sector and provided a path forward for ways in which NIST and other national measurement laboratories and standards organizations can focus their efforts and accelerate the sustainable commercialization of CNM. A key finding was that rapid and highresolution size, morphology and chemistry characterization tools must be designed and implemented to enable this exciting new material to be fully commercialized.

\section{Potential characterization techniques}

$\mathrm{CNM}$ has the potential to change the current paradigms of nanotechnology. However, to be able to produce large quantities of CNM, in-line process control characterization techniques still need to be developed. Metrology and standards can accelerate commercialization. The authors suggest that the following measurements be considered as potential tools to track in-line processing since they have a good balance between measurement sensitivity and speed of measurement.

- Nuclear magnetic resonance (NMR) spectroscopy: this tool can be adapted to perform high-throughput, continuous measurements that allow for the detection of surfacebound water. ${ }^{31}$ NMR measurements could possibly allow manufacturers to monitor the degree of chemical surface functionalization as well as the morphology (crystalline versus amorphous) of the cellulose nanoparticles. ${ }^{32}$

- Inverse-gas chromatography (iGC): iGC is useful in measuring the surface energy distribution (due to weak intermolecular forces such as van der Waals, hydrogen bonding, etc.) of powdered or highly porous materials by 
What do we still need to understand to commercialize cellulose nanomaterials? Davis, Grolman, Karim and Gilman measuring the retention times of various gasses through a known volume of material. ${ }^{33-35}$ To determine the surface energy distribution of cellulose nanomaterial, iGC would allow detailed analysis of small batches for quality assurance.

- Fluorescence lifetime spectroscopy, time-correlated single photon counting spectroscopy or frequency-domain lifetime measurements: by monitoring the lifetime of the autofluorescence of lignin attached to the CNM, manufacturers could utilize this technique to determine and maintain the processing conditions that allow for complete removal of lignin, while avoiding overprocessing and degrading the cellulose nanomaterials. ${ }^{36,37}$

- Microwave cavity perturbation: a non-contact, nondestructive microwave-based technique could be adapted to in-line CNM production for monitoring the dielectric constant and water content. ${ }^{38-40}$ This microwave free-space technique is most suitable for monitoring large-area, sheetlike materials such as films and roll-to-roll processes (e.g., paper manufacturing).

While this is by no means an exhaustive list of possible new approaches, it represents some of the most promising, fairly unexplored measurements the authors currently envision. The authors hope that this document will ignite further discussion and inspire the development of other, more creative solutions.

\section{Conclusion}

CNM are of great interest to many materials researchers and engineers in fundamental research (academia and government institutes), industry and the standards community. The potential of $\mathrm{CNM}$ as a sustainable and potentially non-hazardous nanomaterial additive in composite systems, industrial processing fluids and barrier films is currently hindered by the lack of reliable, industrially relevant measurement tools required to control and verify uniformity of $(a)$ geometry, $(b)$ chemistry, $(c)$ processing and $(d)$ environmental health impacts. Naturally derived materials have a much greater inherent variation, due to the various sources from which they are obtained and the manner in which they are processed. Thus, the need for robust measurements is even more critical than for synthetic systems.

CNM morphology and surface characteristics are the two general sets of properties that need to be quantified to address quality control. Morphology encompasses geometric properties such as length, width, shape and aspect ratio. It also refers to degree of crystallinity and polymer chain configuration within CNC. For $\mathrm{CNF}$, morphological concerns such as extent of branching and degree of entanglement also persist. Surface properties that need to be measured include surface chemistry (i.e., surface sulfur content for wood-derived, $\mathrm{H}_{2} \mathrm{SO}_{4}$-processed $\mathrm{CNM}$ ), surface energy distribution and dispersibility in various media.
While these general properties must be measured for development of commercial applications, several other measurement requirements have to be addressed before full-scale industrial production of CNM can occur. Quality measurements need to be affordable, in-line, high-throughput and easy to use to facilitate manufacturing process monitoring. Many of the current techniques used to quantify these key properties are too expensive. Those of us working with this promising nanomaterial have a unique opportunity to expedite commercialization by developing the appropriate tools that meet the measurement needs mentioned above. The authors have only proposed a handful of potential solutions and eagerly anticipate the characterization techniques that will be invented and implemented as the cellulose nanomaterial industry matures.

\section{REFERENCES}

1. Huang, J.; Zhu, H.; Chen, Y.; Preston, C.; Rohrbach, K.; Cumings, J.; Hu, L. Highly transparent and flexible nanopaper transistors. ACS Nano 2013, 7, 2106-2113.

2. Ummartyotin, S.; Juntaro, J.; Sain, M.; Manuspiya, $\mathrm{H}$. Development of transparent bacterial cellulose nanocomposite film as substrate for flexible organic light emitting diode (OLED) display. Industrial Crops and Products 2012, 35, 92-97.

3. Zhu, H.; Xiao, Z.; Liu, D.; Li, Y.; Weadock, N. J.; Fang, Z.; Huang, J.; Hu, L. Biodegradable transparent substrates for flexible organic-light-emitting diodes. Energy Environ. Sci. 2013, 6, 2105-2111.

4. He, X.; Xiao, Q.; Lu, C.; Wang, Y.; Zhang, X.; Zhao, J.; Zhang, W.; Zhang, X.; Deng, Y. Uniaxially aligned electrospun all-cellulose nanocomposite nanofibers reinforced with cellulose nanocrystals: scaffold for tissue engineering. Biomacromolecules 2014, 15, 618-627.

5. Domingues, R. M. A.; Gomes, M. E.; Reis, R. L. The potential of cellulose nanocrystals in tissue engineering strategies. Biomacromolecules 2014, 15, 2327-2346.

6. Utsel, S.; Malmström, E.; Carlmark, A.; Wågberg, L. Thermoresponsive nanocomposites from multilayers of nanofibrillated cellulose and specially designed $\mathrm{N}$-isopropylacrylamide based polymers. Soft Matter 2010, 6 , 342-352.

7. Moon, R. J.; Martini, A.; Nairn, J.; Simonsen, J.; Youngblood, J. P. Cellulose nanomaterials review: structure, properties and nanocomposites. Chemical Society Reviews 2011, 40, 3941-3994.

8. Biyani, M. V.; Jorfi, M.; Weder, C.; Foster, E. J. Lightstimulated mechanically switchable, photopatternable cellulose nanocomposites. Polymer Chemistry 2014, 5 , 5716-5724.

9. Zammarano, M.; Maupin, P. H.; Sung, L.-P.; Gilman, J. W.; McCarthy, E. D.; Kim, Y. S.; Fox, D. M. Revealing the interface in polymer nanocomposites. ACS Nano 2011, 5, 3391-3399.

10. Zhang, Z.; Sèbe, G.; Rentsch, D.; Zimmermann, T.; Tingaut, P. Ultralightweight and flexible silylated nanocellulose sponges 
for the selective removal of oil from water. Chemistry of Materials 2014, 26, 2659-2668.

11. Mukherjee, S. M.; Woods, H. J. X-ray and electron microscope studies of the degradation of cellulose by sulphuric acid. Biochimica et Biophysica Acta 1953, 10, 499-511.

12. Turbak, A. F.; Snyder, F. W.; Sandberg, K. R. Microfibrillated cellulose Paper $360^{\circ}$-TAPPI 1983, 4374702.

13. Walker, $C$. Thinking small is leading to big changes. Paper $360^{\circ}$-TAPPI 2012, 40, 8-13.

14. Health Canada. 8. List of Permitted Food Additives with Other Generally Accepted Uses (Lists of Permitted Food Additives). See http://www.hc-sc.gc.ca/fn-an/securit/addit/list/8-otherautre-eng.php (accessed 01/01/2015).

15. Sinko, R.; Qin, X.; Keten, S. Interfacial mechanics of cellulose nanocrystals. MRS Bulletin 2015, 40, 340-348.

16. Wise, L. E.; Murphy, M.; D'Addieco, A. A. Chlorite holocellulose, its fractionation and bearing on summative wood analysis and on studies on the hemicelluloses. Paper $360^{\circ}$-TAPPI 1946, 29, 210-218.

17. Isogai, A.; Kato, Y. Preparation of polyuronic acid from cellulose by TEMPO-mediated oxidation. Cellulose 1998, 5, 153-164.

18. Siró, I.; Plackett, D. Microfibrillated cellulose and new nanocomposite materials: a review. Cellulose 2010, 17, 459-494.

19. Wu, X.; Moon, R. J.; Martini, A. Crystalline cellulose elastic modulus predicted by atomistic models of uniform deformation and nanoscale indentation. Cellulose 2012, 20 , $43-55$.

20. Mariano, M.; El Kissi, N.; Dufresne, A. Cellulose nanocrystals and related nanocomposites: review of some properties and challenges. Journal of Polymer Science Part B: Polymer Physics 2014, 52, 791-806.

21. Sacui, I. A.; Nieuwendaal, R. C.; Burnett, D. J.; Stranick, S. J.; Jorfi, M.; Weder, C.; Foster, E. J.; Olsson, R. T.; Gilman, J. $\mathrm{W}$. Comparison of the properties of cellulose nanocrystals and cellulose nanofibrils isolated from bacteria, tunicate, and wood processed using acid, enzymatic, mechanical, and oxidative methods. ACS Applied Materials \& Interfaces 2014, 6, 6127-6138.

22. Dri, F. L.; Hector, L. G.; Moon, R. J.; Zavattieri, P. D. Anisotropy of the elastic properties of crystalline cellulose I $\beta$ from first principles density functional theory with van der Waals interactions. Cellulose 2013, 20, 2703-2718.

23. Diaz, J. A.; Wu, X.; Martini, A.; Youngblood, J. P.; Moon, R. $J$. Thermal expansion of self-organized and shear-oriented cellulose nanocrystal films. Biomacromolecules 2013, 14 , 2900-2908.

24. Pan, J.; Hamad, W.; Straus, S. K. Parameters affecting the chiral nematic phase of nanocrystalline cellulose films. Macromolecules 2010, 43, 3851-3858.
25. Dumanli, A. G.; van der Kooij, H. M.; Kamita, G.; Reisner, E.; Baumberg, J. J.; Steiner, U.; Vignolini, S. Digital color in cellulose nanocrystal films. ACS Applied Materials \& Interfaces 2014, 6, 12302-12306.

26. Gardner, D. J.; Oporto, G. S.; Mills, R.; Azizi Samir, M. A. S. Adhesion and surface issues in cellulose and nanocellulose. Journal of Adhesion Science and Technology 2008, 22, 545-567.

27. Shatkin, J. A.; Wegner, T. H.; Bilek, E. M.; Cowie, J. Market projections of cellulose nanomaterial-enabled products - part 1: applications. TAPPI Journal 2014, 13, 9-16.

28. Davis, C. S.; Moon, R. J.; Ireland, S.; Johnston, L.; Shatkin, J. A.; Nelson, K.; Foster, E. J.; Forster, A. M.; Postek, M. T.; Vladar, A.; Gilman, J. W. NIST-TAPPI Workshop on Measurement Needs for Cellulose Nanomaterial, NIST SP 1192. Gaithersburg, MD, USA: National Institute for Standards and Technology, 2015.

29. Boluk, Y.; Moon, R.; Ensor, D.; Nieh, W.; Forsstrom, U.; Shatkin, J. A.; Gardner, D. J.; Teague, C.; Haydon, B.; Walker, C.; Willis, C.; Wegner, T. H. Roadmap for the Development of International Standards for Nanocellulose. TAPPA NanoDivision: Peachtree Corners, GA, USA, 2011.

30. Canadian Standards Association. Cellulosic Nanomaterials - Test Methods for Characterization. Ontario, Canada: Canadian Standards Association, 2014, Z5100-14.

31. Fairhurst, D.; Cosgrove, T.; Prescott, S. W. Relaxation NMR as a tool to study the dispersion and formulation behavior of nanostructured carbon materials. Magnetic Resonance in Chemistry 2015, DOI: 10.1002/mrc.4218.

32. Atalla, R. H.; Vanderhart, D. L. The role of solid state 13C NMR spectroscopy in studies of the nature of native celluloses. Solid State Nuclear Magnetic Resonance 1999, 15, $1-19$.

33. Mills, R. H.; Tze, W. T. Y.; Gardner, D. J.; Heiningen, A. V. Inverse gas chromatography for the determination of the dispersive surface free energy and acid - base interactions of a sheet molding compound. I. Matrix material and glass. Journal of Applied Polymer Science 2008, 109, 3519-3524.

34. Mills, R. H.; Gardner, D. J.; Wimmer, R. Inverse gas chromatography for determining the dispersive surface free energy and acid-base interactions of sheet molding compound-part II 14 ligno-cellulosic fiber types for possible composite reinforcement. Journal of Applied Polymer Science 2008, 110, 3880-3888.

35. Peng, Y.; Gardner, D. J.; Han, Y.; Cai, Z.; Tshabalala, M. A. Influence of drying method on the surface energy of cellulose nanofibrils determined by inverse gas chromatography. Journal of Colloid and Interface Science 2013, 405, 85-95.

36. Coletta, V. C.; Rezende, C. A.; Conceição, F. R. D.; Polikarpov, I.; Guimarães, F. E. G.; Rodrigues, F.; Polikarpov, I.; Eduardo, F.; Guimarães, G. Mapping the lignin distribution in pretreated sugarcane bagasse by confocal and fluorescence lifetime 
imaging microscopy. Biotechnology for Biofuels 2013, 6 , 43-52.

37. Chimenez, T. A.; Gehlen, M. H.; Marabezi, K.; Curvelo, A. A. $\mathrm{S}$. Characterization of sugarcane bagasse by autofluorescence microscopy. Cellulose 2014, 21, 653-664.

38. Wee, F. H. H.; Soh, P. J. J.; Suhaizal, A. H. M. H. M.; Nornikman, H.; Ezanuddin, A. A. M. A. M. Free space measurement technique on dielectric properties of agricultural residues at microwave frequencies, Microwave and Optoelectronics Conference (IMOC), 2009 SBMO/IEEE MTT-S International, Belem, Brazil, 2009, I, 183-187.
39. Malik, S. A.; Ghodgaonkar, D. K.; Majid, W. M. W. A.; Nuruddin, M. F. A study on moisture content variation of Malaysian wood using microwave nondestructive testing at 8 to $12 \mathrm{GHz}$, Proceedings of URSI Conference, New Delhi, India, 2005, 23-29.

40. Aris, M. A. B.; Ghodgaonkar, D. K.; Khadri, N. Nondestructive and noncontact dielectric measurement methods for low-loss liquids using free space microwave measurement system in $8-12.5 \mathrm{GHz}$ frequency range, $2004 R F$ and Microwave Conference (IEEE Cat. No.04EX924), Subang, Malaysia, 2004, 182-189.
WHAT DO YOU THINK?

To discuss this paper, please email up to 500 words to the managing editor at gmat@icepublishing.com

Your contribution will be forwarded to the author(s) for a reply and, if considered appropriate by the editor-inchief, will be published as a discussion in a future issue of the journal.

ICE Science journals rely entirely on contributions sent in by professionals, academics and students coming from the field of materials science and engineering. Articles should be within 5000-7000 words long (short communications and opinion articles should be within 2000 words long), with adequate illustrations and references. To access our author guidelines and how to submit your paper, please refer to the journal website at www.icevirtuallibrary.com/gmat 